\title{
CONTROL OF INTERFACIAL EQUILIBRIA BY LOCAL CHARGE DISTRIBUTION AND AVERAGE SURFACE POTENTIAL
}

\author{
DIETMAR MÖBIUS, WOLFGANG CORDROCH, ROLF LOSCHEK, LI FENG CHI, ARUNA \\ DHATHATHREYAN AND VIOLA VOGEL \\ Max-Planck-Institut für biophysikalische Chemie (Karl-Friedrich-Bonhoeffer-Institut), Postfach 284I, \\ D-3400 Göttingen (F.R.G.)
}

(Received April 25, 1989; accepted June 9, 1989)

The incorporation of cadmium ions in porphyrins embedded in matrix monolayers is dominated by head group interactions as opposed to the effect of the average surface potential on interfacial cation concentrations. Similar phenomena are also observed in the adsorption of water-soluble porphyrin complexes to spread monolayers. The influence of such local interactions can be eliminated when the composition of the head group region is kept constant and the interfacial potential is modified by changing dipole layers at distances of about $2.5 \mathrm{~nm}$ from the head group interface. Protonation equilibria can be systematically shifted by planned variation of the average potential via such remote dipole layers.

\section{INTRODUCTION}

The influence of the surface potential on equilibria at interfaces such as the solid-liquid interface is usually described by the Gouy-Chapman-Stern theory. Potential probes show changes in their spectroscopic properties when the interfacial potential changes (see for example ref. 1). However, local interactions, i.e. the effect of local charge distribution, may dominate the effect of the average potential on adsorption and chemical equilibria, as obvious in biocatalysis. Organized monolayers at the air-water or the assembly-water interface are excellent model structures for systematic variation of the important parameters in order to elucidate the role of the different influences on equilibria.

A monolayer has two interfaces to the nearest environment: the surface of hydrophobic end groups and the surface with hydrophilic head groups. Both interfaces contribute to the interfacial potential. The surface potential of a monolayer on water is usually given as the difference $\Delta V$ between the surface potentials of the monolayer-covered water surface and of the clean water surface. A separation of the measured $\Delta V$ into two components has recently been achieved ${ }^{2}$. The component of the monolayer-air interface can be given as absolute potential, whereas the second component contains the contribution of the electric double layer and all other contributions (e.g. the influence of a different water structure in contact with the monolayer as compared with the clean water surface). The 
interfacial potential may be varied systematically by using either amphiphiles with different head groups or amphiphiles with the same head group and modified hydrophobic chains. A change of the composition of the head group region may change equilibria of a probe molecule via modification of local interactions and charge distribution in the near environment. Such a change of the near environment of a probe molecule in the head group region can be avoided by modifying the dipole layer constituted by the hydrophobic end groups of the dense-packed amphiphiles. Some examples of the two strategies are presented without describing experimental details, which are given in the referenced publications or will be published separately.

\section{EFFECTS OF LOCAL CHARGE DISTRIBUTION}

\subsection{Interfacial complex equilibria}

Porphyrins form metal complexes with divalent ions such as $\mathrm{Cd}^{2+}$ by substitution of the two protons in the centre of the porphine ring (for the porphine structure see Fig. 1). Therefore the complex equilibrium depends on the protonation equilibria, and shifts in the value of $p K_{\mathrm{a}}$ influence the complex equilibrium constant $K_{\mathrm{c}}$. The complex equilibria of two amphiphilic porphyrins, a cation with four positive charges at the periphery of the ring, APC (see Fig. 3 for its structure), and a neutral porphyrin, APN, have been investigated in mixed monolayers at the air-water interface ${ }^{3,4}$.

A change in the surface potential causes a change in the interfacial concentrations of both $\mathrm{H}^{+}$and $\mathrm{Cd}^{2+}$ as described by the Gouy-Chapman theory such that the competition of these cations for the centre of the porphyrin is not influenced. Therefore the complex equilibrium should not be shifted when the monolayer matrix is changed, provided that the intrinsic $p K$ of the porphyrin remains constant. This assumption may not be valid, as shown recently for a fluorescent probe embedded in neutral monolayers ${ }^{5}$. The amphiphilic porphyrin APC exhibited a strong shift of the complex equilibrium to more complex when the matrix was changed from eicosanol to arachidic acid ${ }^{3}$. This fact points to the dominant influence of specific interactions of the cadmium ions with arachidic acid molecules that fill the space on top of the flat-lying porphyrin ring.

The role of local charge distribution was demonstrated more strikingly by comparing the complex equilibria of the porphyrin APC in a monolayer matrix with that of a neutral amphiphilic porphyrin APN. The matrix of the APN monolayer was composed such that the surface potentials of both porphyrin monolayers were matched ${ }^{3}$. The equilibrium of the cationic porphyrin was considerably displaced to more complex with respect to that of the neutral porphyrin.

\subsection{Adsorption phenomena}

Another example of the influence of local interactions is the adsorption of water-soluble porphyrins and their cadmium complexes to different monolayers ${ }^{4}$. The anionic porphyrin SPA can be adsorbed from the aqueous subphase to a monolayer containing positively charged head groups; see Fig. 1(a). This is found by measuring the reflection spectrum $\Delta R$ (full curve in Fig. 1(a)), since the molecules 
in solution do not contribute to the signal ${ }^{6} ; \Delta R$ is the difference in reflectivity between the monolayer-covered surface and the clean water surface. Only the free base porphyrin (band at $415 \mathrm{~nm}$ ) is adsorbed although the cadmium complex (band at $435 \mathrm{~nm}$ ) is the dominant species in the bulk solution as evidenced by the absorption spectrum $A$ (broken curve in Fig. 1(a)).

In the case of the cationic porphyrin SPC the cadmium complex (band at $465 \mathrm{~nm}$ ) is much more strongly bound to the monolayer of arachidic acid than the free base; see Fig. 1(b), full curve ( $\Delta R)$. In the bulk, only the free base is observed as evidenced by the absorption spectrum $A$ (band at $420 \mathrm{~nm}$; broken curve) in Fig. 1(b). These drastic differences in binding of the different species to the two monolayers cannot be explained as a consequence of the different average surface potentials but must be due to specific interactions.
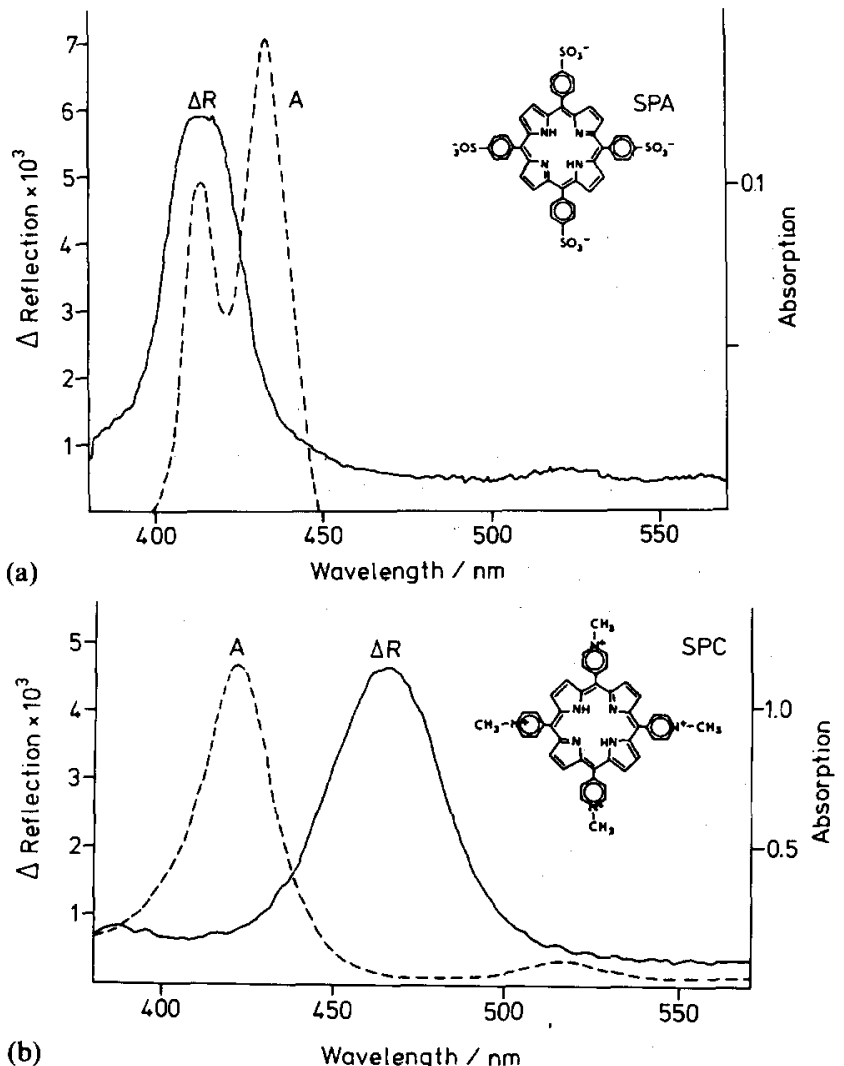

Fig. 1. Adsorption of water-soluble porphyrins and their cadmium complexes from the aqueous subphase to monolayers; reflection spectra $(-, \Delta R)$ from the air-water interface and absorption spectra of the aqueous subphase $(---, A)$. (a) Mixed monolayer of eicosylamine and methyl arachidate, molar ratio 2:3; surface pressure $20 \mathrm{mN} \mathrm{m}^{-1}$; subphase $5 \times 10^{-7} \mathrm{M}$ porphyrin $\mathrm{SPA}, 10^{-3} \mathrm{M} \mathrm{CdCl}_{2}$ and $5 \times 10^{-5} \mathrm{M} \mathrm{NaHCO}_{3}$; preferential adsorption of the free base (band at $415 \mathrm{~nm}$, reflection spectrum), although in subphase mainly complex (band at $435 \mathrm{~nm}$, absorption spectrum). (b) Monolayer of arachidic acid; surface pressure $20 \mathrm{mN} \mathrm{m}^{-1}$; subphase $5 \times 10^{-6} \mathrm{M}$ porphyrin $\mathrm{SPC}$ and $10^{-3} \mathrm{MCdCl}_{2}$; preferential adsorption of the complex (band at $465 \mathrm{~nm}$, reflection spectrum), although in subphase free base only (band at $420 \mathrm{~nm}$, absorption spectrum). 


\section{EFFECTS OF AVERAGE INTERFACIAL POTENTIAL}

The contribution of the interface (methyl groups)-air to the total surface potential of dense-packed monolayers is $+665 \mathrm{mV}$ (positive pole pointing to the air). If the methyl groups are replaced by trifluoro-methyl groups this contribution is $-605 \mathrm{mV}$. Since palmitic acid and $\omega$-trifluoro-stearic acid form mixed monolayers at the air-water interface ${ }^{7}$, the surface potential of a matrix monolayer can be varied systematically by mixing various fractions of these acids. The particularly interesting feature of such mixtures is the fact that the composition of the head group region is maintained constant. Only dipole layers separated by about $2.5 \mathrm{~nm}$ from the location of a probe are changed.

\subsection{Potential probe at the air-water interface}

Various cyanine dyes have been used as potential probes in microheterogeneous systems and membranes since their fluorescence depends on the interfacial or transmembrane potential. Different mechanisms for this behaviour have been proposed ${ }^{8}$. The surface potential of the matrix for the amphiphilic cyanine dye CY (see Fig. 2 for its structure) was varied according to the strategy described above using mixed monolayers of palmitic acid and $\omega$-trifluoro-stearic acid. The chromophore (molar fraction of dye 0.01) which is located at the hydrophilic interface of the monolayer on water experiences no change in the composition of the near environment.

The relative fluorescence intensity of the probe at $515 \mathrm{~nm}$ is plotted in Fig. 2 against the surface potential of the matrix monolayers ${ }^{7}$. A clear dependence of the fluorescence intensity (no change in the spectrum with variation of the matrix) on the average surface potential is seen. From reflection measurements it is concluded that the effect may be due to a potential-dependent association of the chromophores to dimers ${ }^{7}$. A more detailed explanation of how the average surface potential influences dye association cannot be given yet.

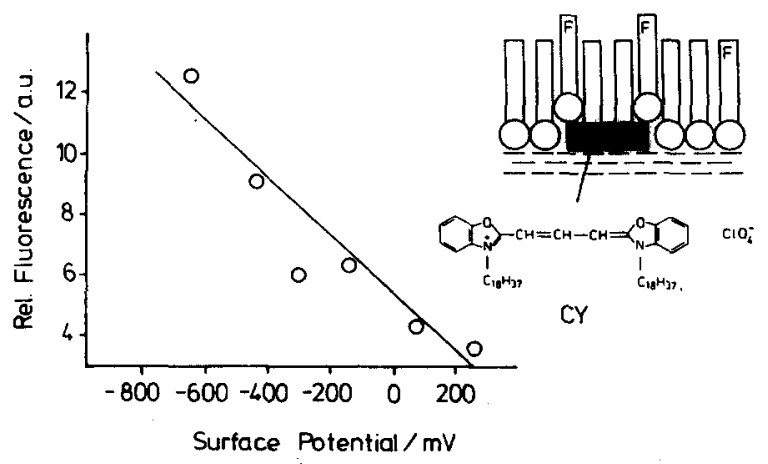

Fig. 2. Response of a fluorescent potential probe to variation of the surface potential by modification of a dipole layer separated from the interface by $2.5 \mathrm{~nm}$; cyanine dye $\mathrm{CY}$ in mixed monolayers of palmitic acid and $\omega$-trifluoro-stearic acid of varying molar ratios at the air-water interface; plot of fluorescence intensity at $515 \mathrm{~nm}$ against surface potential of the monolayer matrix; strong dependence of fluorescence yield on the surface potential, although the near environment of the probe is not changed. 


\subsection{Protonation equilibria in transferred monolayers}

A compound used as potential probe ${ }^{8}$ and also for the investigation of nonlinear optical phenomena ${ }^{9}$ is the alkylaminostyryl-pyridin chromophore ASP. This compound is water soluble when the alkyl substituents are short and forms stable monolayers with one or two long alkyl chains ${ }^{10}$. In aqueous solutions the dimethylASP is neutral at $\mathrm{pH} 7$, takes up one proton at $\mathrm{pH} 5.6$ and exists as a dication at $\mathrm{pH} 2$. These forms are characterized by their different absorption spectra, with maxima at $325 \mathrm{~nm}(\mathrm{pH} 2), 440 \mathrm{~nm}(\mathrm{pH} 5.6)$ and $400 \mathrm{~nm}(\mathrm{pH} 7)$.

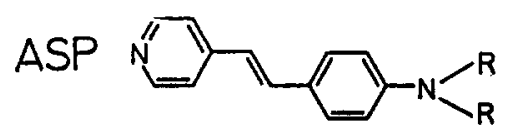

The octadecyl-ASP forms monolayers; however, owing to chromophore association these monolayers are extremely rigid. Mixed monolayers of C18-ASP and arachidic acid, molar ratio $1: 5$, can easily be transferred to solid substrates with the usual LB technique ${ }^{10}$. In the mixed monolayer on water, C18-ASP is protonated to the monocationic form as shown by a band at $440 \mathrm{~nm}$ in the reflection spectrum. After transfer to a hydrophobic glass plate and coating with a protective monolayer of arachidic acid the C18-ASP shows the spectrum of the dication form. This shift of the protonation equilibrium may be due to a change in both the interfacial potential and local interactions upon transfer of the protective monolayer. Further, the average orientation of the transition moment has changed. In the mixed monolayer on water a spatially random orientation is observed, whereas in the transferred monolayer the transition moments have an average angle of $29^{\circ}$ with respect to the surface normal ${ }^{10}$. The protonation equilibrium of the C18-ASP in monolayer assemblies can be reversibly shifted by exposure to $\mathrm{NH}_{3}$ vapour followed by $\mathrm{HCl}$ vapour, without change in the average chromophore orientation and noticeable degradation of the assembly.

\subsection{Effect of remote dipole orientation}

In monolayer organizates on glass plates the average surface potential may be varied by changing remote dipole layers by deposition of different sublayers underneath the monolayer that remains in contact with the aqueous phase. The protonation equilibrium of the amphiphilic porphyrin APC in mixed monolayers has been studied in such a way ${ }^{11}$.

Figure 3 shows a logarithmic plot of the ratio of free base to protonated form as determined from the measured transmission spectra against the bulk $\mathrm{pH}$ of the adjacent aqueous phase. The data obtained from reflection measurements for spread monolayers at the air-water interface (crosses, full line 1) are given for reference. When the mixed APC monolayer is directly transferred to a hydrophobic glass plate (see squares in Fig. 3) only small deviations from line 1 are observed. Transfer of the APC monolayer on a glass plate coated with eisosylamine causes a considerable shift of the protonation equilibrium to a smaller $p K$ value (circles, broken line 2). The interfacial concentration of $\mathrm{H}^{+}$is obviously diminished by the field of the positive ammonium head groups of the underlying monolayer. A 


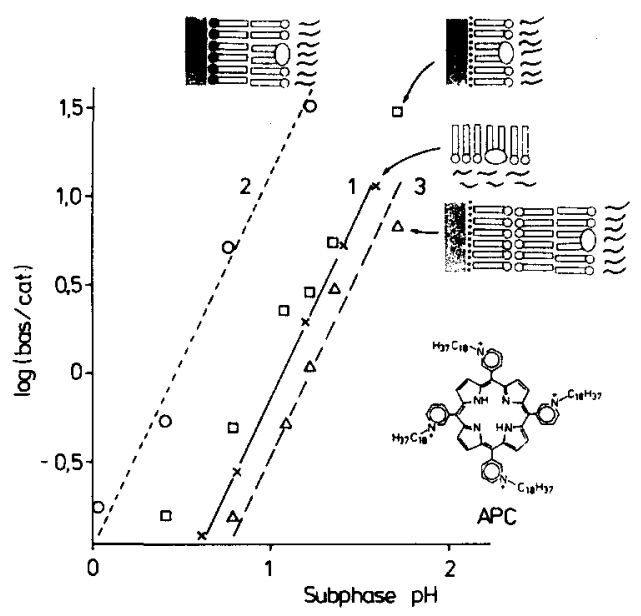

Fig. 3. Shift of interfacial protonation equilibria of the porphyrin APC in transferred monolayers in contact with the aqueous phase by variation of underlying monolayer assembly (architecture shown schematically); logarithmic plot of the ratio free base to protonated form as determined spectroscopically against $\mathrm{pH}$ of the adjacent aqueous phase; $\mathrm{x}-$, line 1, equilibrium at the air-water interface (for reference); $\square$, APC monolayer transferred to hydrophobic glass plates; $\bigcirc---$, line 2, APC monolayer transferred to a monolayer of eicosylamine deposited onto glass plates; strong shift of the equilibrium to smaller $p K_{\mathrm{a}} ; \triangle---$, line 3, APC monolayer transferred to fatty acid bilayer on hydrophobic glass plates; small shift of the equilibrium to larger $p K_{\mathrm{a}}$; APC monolayer; mixed APC and arachidic acid, molar ratio $1: 20$.

controversial effect is seen when the sublayer consists of two fatty acid monolayers deposited onto a hydrophobic glass plate (triangles, broken line 3$)^{11}$.

The porphyrin APC provides a particular advantage as a probe. A shift in the intrinsic $p K_{\mathrm{a}}$ of the porphyrin in these experiments can be detected by determining the complex equilibrium constant. If the field of the remote dipole layers influences the interfacial $\mathrm{pH}$ only without modifying the intrinsic $p K_{\mathrm{a}}$ or $p K_{\mathrm{c}}$ (no change in the near environment), the ratio of complex to free base should be independent of the structure and composition of the sublayer. This has indeed been found with mixed monolayers of APC and arachidic acid ${ }^{11}$.

A more systematic approach is the investigation of the effect of directional inversion of a dipole layer in monolayer organizates on the protonation equilibrium of a probe ${ }^{12}$. The frequently used fluorescent probe HHC (see Fig. 4 for its structure) was incorporated in a monolayer of arachidic acid (AA), molar ratio $1: 400$, and deposited on top of a monolayer of AA on glass. The triangles (full line 2) in Fig. 4 show a logarithmic plot of $\alpha /(1-\alpha)$, where $\alpha$ is the dissociation degree of the probe molecule against the bulk $\mathrm{pH}$ of the adjacent aqueous phase. The intercept of the straight line with the horizontal line at $\lg \{\alpha /(1-\alpha)\}=0$ yields the value of $p K_{\mathrm{a}}=10.2$.

Incorporating the $\mathrm{HHC}$ in a matrix monolayer of palmitic acid and $\omega$ trifluorostearic acid, molar ratio 7:3, shifts the protonation equilibrium to smaller $p K_{\mathrm{a}}$ as shown by the circles in Fig. 4 (direction of dipoles indicated). From the broken line 1 the value of $p K_{\mathrm{a}}=9.5$ is obtained. A shift in this direction is expected 
since the positive end of the dipoles constituted by the fluorocarbon end groups of the outer monolayer points to the aqueous phase. Consequently, a higher bulk $\mathrm{H}^{+}$ concentration is required for half-protonation as compared with the former situation.

The opposite effect should be observed when the direction of the dipoles is reversed. The experimental data shown in Fig. 4 (squares, broken line 3) clearly provide evidence for such an effect. The underlying monolayer in contact with the

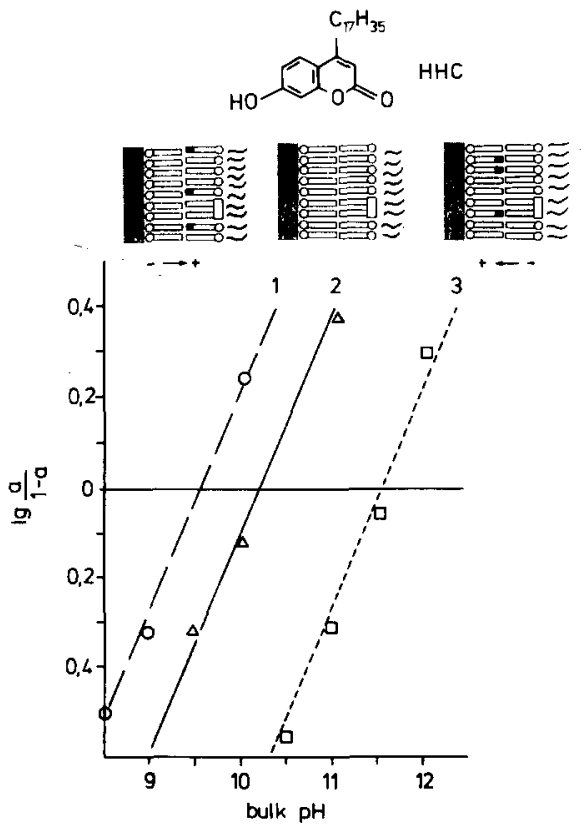

Fig. 4. Influence of inversion of a remote dipole layer in a monolayer assembly on glass plates on the protonation equilibrium of the fluorescent probe $\mathrm{HHC}$ embedded in a matrix monolayer (assembly architecture shown schematically); $\triangle-$, line 2, mixed monolayer of $\mathrm{HHC}$ and arachidic acid, molar ratio 1:400, deposited onto a monolayer of arachidic acid (AA) on glass, $p K_{\mathrm{a}}=10.2 ; \mathrm{O}---$, line 1 , monolayer of HHC, palmitic acid (PA) and w-trifluoro-stearic acid (FSA), molar ratio 1:120:280, deposited onto a monolayer of AA on glass, $p K_{\mathrm{a}}=9.5$; shift of the $p K$ due to orientation of the dipoles of FSA hydrophobic ends with the positive pole to the aqueous phase; $\square---$, line 3 , monolayer of $\mathrm{HHC}$ and AA, molar ratio $1: 400$, deposited onto a mixed monolayer of PA and FSA, molar ratio $7: 3$, on glass, $p K_{\mathrm{a}}=11.55$; opposite $p K$ shift due to inverse orientation of the FSA hydrophobic end dipoles.

glass is now composed of palmitic acid and $\omega$-trifluoro-stearic acid, molar ratio 7:3, and the HHC is embedded in a monolayer of arachidic acid. The value of $p K_{\mathrm{a}}=11.55$ can be taken from the intercept. The negative end of the fluorocarbon dipoles points to the adjacent aqueous phase enhancing the interfacial $\mathrm{H}^{+}$ concentration.

\section{CONCLUSIONS}

The monolayer technique provides unique possibilities of varying systemati- 
cally the interfacial potential. A change in the near environment of a probe molecule may modify the intrinsic properties of the probe in addition to the effect on interfacial ion concentrations. The shifts of complex equilibria described in Section 2 show clearly that the evaluation of interfacial ion concentrations or interfacial potentials by applying standard theories is questionable. The effect of remote dipole layers that are organized in a planned way, however, is restricted to the electrostatic action without change of the near environment of a probe. Therefore the intrinsic $p K$ of the probe remains constant when remote dipole layers are varied, as shown in an example in Section 3. On this basis, the response of potential probes can now be calibrated.

\section{ACKNOWLEDGMENT}

This work was supported by the Bundesministerium für Forschung und Technologie.

\section{REFERENCES}

E. Grell (ed.), Membrane Spectroscopy, Springer-Verlag, Berlin, 1981.

2 V. Vogel and D. Möbius, J. Colloid Interface Sci., 126 (1988) 408.

3 R. Loschek and D. Möbius, Chem. Phys. Lett., 151 (1988) 176.

4 R. Loschek and D. Möbius, J. Chim. Phys., 85 (1988) 1041.

5 J. G. Petrov and D. Möbius, Langmuir, 5 (1989) 523.

6 H. Grüniger, D. Möbius and H. Meyer, J. Chem. Phys., 79 (1983) 3701.

7 W. Cordroch, V. Vogel and D. Möbius, submitted to J. Chem. Phys.

8 A. S. Waggoner, Ann. Rev. Biophys. Bioenerg., 8 (1979) 847.

9 R. Steinhoff, L. F. Chi, G. Marowsky and D. Möbius, J. Opt. Soc. Am. B, $6(1989) 843$.

10 L. F. Chi, A. Dhathathreyan and D. Möbius, submitted to Langmuir.

11 R. Loschek and D. Möbius, to be published.

12 W. Cordroch and D. Möbius, submitted to J. Phys. Chem. 